Rev. Chil. Pediatr. 68 (1); $11-15,1997$

\title{
Crecimiento de niños en zonas rurales
}

\author{
Patricia Bustos M. ${ }^{\text {; }}$ Hugo Amigo C. ${ }^{\text {; }}$; \\ Elena Bustos D.'
}

\begin{abstract}
Resumen
El propósito de este estudio fue analizar la evclución del crecimiento en estatura de escolores de ralio boja. Mediante rastreo. en escolares de seis o ccho años de zonas furales de alio vulnerobilidad social de las Regiones ivetrapoliana y Sexta, de Chle, se seleccionaron 127 niños con déficis de lalla para la edad entre menos 1 y menos 3 funlajes $Z$ de la mediana del parón NCHS/OMS (casosi y 130 conlroles cuya es:alura estaba entre más o menos medio puniaje Z. Se analizó la información de las fichas de los consultorios donde eran controlados. Se abservó un incremento menor de estatura en los casos ontes y después de la edad de 24 meses $p<<0,0011$. La mayor des. aceleración de a talla ocurrió en el primer año de vida ios cosos mostraban valores inferiores de punkajes Z '-0,05 ws $-0,13\}$ desce el nacimiento. llegando a $-1,72$ a los 18 meses y a $-1,83$ a los 72 meses. Los controles se mantuvieron alrededor de la mediana durante este mismo periodo. La desnutrición y lalla baja al nacer se asocioron significativamente con taila baja en el escóar iventaja $4 O R=4,94$ y 3,36 respectivamentel. Estos resultados sugiéen a conveniencia de reforzor les acciones de prevención y control prenatales y posnatales si se desea disminuir lo trecuencia de tallo baja en los escolares chilenos.
\end{abstract}

(Palabra clave: crecimien:c.)

\section{Growth of children from some chilean rural areas}

To analize height growth olong the firsl vears of life in rutal chi'ean children, 127 stunted schood children (cases; aged 6 To 8 yesar old were compored to 130 normal icontro) children of the same age, both living in rural oreas of high sorial vunerasility at centrol Chile. Coses were selecled by a height for oge index (NCHS/WHOi between -7 and -32 scores and controls by height for age index within $\pm 0.5 \mathrm{Z}$ scores. Anthropometric records were obtained fron well baby and child health conirol fites and reirospectively analyzed. Stunted children showed significonily lower height for age Z score tian control children cl birth $\{-0.05$ versus -0.13 ) ard lower height increases both before and after age 2.4 months ip $<0.00$ ] porticularily alang the hirst year of life, but also at 18 month [Z scores -1.72 ] and of 72 monith :-1.831. Control children's height $z$ scores were close to median values of the NCHS/WHO's standard al the correspondirg oges. Stunted schookhildren exhibited higher odds for undernulrition and small size at birth lodds ralic $=4.94$ and 3.36 , respecivelyl). Prevention of small size at bisth and underrutrition during infancy may both be efiecive meassures to reduce height stunting among chileon scinool children.

[Key word: growih.)

Un aspecto relevante de la pediatría es su preocupación por el crecimiento y desarrollo. El personal de salud que atiende a la población infantil debe estar familiarizado con los criterios de normalidad, para reconocer precozmente des-

1. Departamento de Nutrición, Facultad de Medicina, Universidad de Chile.

Financiado por Proyecto Fondecyt 919-92. viaciones de lo esperado, identificar el problema subyacente y brindar a tiempo un tratamiento adecuado.

El retardo de crecimiento es un problema frecuente en niños de los países menos desarrollados, especialmente en lugares en que se concentra la pobreza o donde habita la población indígena, en algunos de los cuales los retardos moderados o graves (déficit que supera las dos desviaciones estándares') afectan a $40 \%$ de los 
niños. En Chile el retardo de crecimiento (superior a una desviación estándar) afecta a un cuarto de la población que ingresa a primer año de la enseñanza básica y su prevalencia es aún mayor en regiones de alta vulnerabilidad social, en que alcanza a la mitad de ellos ${ }^{2}$.

La estatura de los escolares que ingresan a la escuela reflejaría su historia nutricional y de salud, por to que se ha propuesto utilizarla como un indicador de desarrollo socioeconómico ${ }^{3,4}$. Frecuentemente, el déficit de talla se detecta cuando la desaceleración del crecimiento ya sc ha producido y es irreversible, por lo tanto es interesante conocer en qué momento se inicia y cuál es su evolución posterior, con la finalidad de prevenirlo o corregirlo oportunamente. La importancia del registro de la estatura radica en que no sólo mide la situación de una determinada población infantil sino, también, porque el déficit se ha asociado a consecuencias adversas, entre ellas rendimiento escolar inferior y menor capacidad física de trabajo en la edad adulta ${ }^{5,6}$.

El objetivo de este trabajo fue pesquisar en niños de talla baja el momento en que se inicia el déficit de estatura y seguir su posterior evolución hasta el comienzo de la edad escolar. La hipólesis que se formuló fue que el déficit observado ocurre fundamentalmente antes de los dos años de vida.

\section{Personas y Métodos}

En una primera fase se hizo un rastreo entre los escolares en cuatro comunas rurales de alta vulnerabitidad social de las Regiones Metropolitana y Sexta para constituir dos grupos: uno de talla baja (casos) y otro normal (controles). Postetiormente se realizó una entrevista domiciliaria y revisaron los registros clínicos de los niños seleccionados. Los métodos se describieron en publicaciones previas ${ }^{7.9}$.

Se identificaron como casos 127 escolares de seis a ocho años con déficit de estatura para la cdad (talla entre menos 1 y menos 3 puntaje $Z$ de la mediana del patrón antropométrico NCHS/ OMS $^{\text {to }}$ y como controles 130 niños de las mis. mas edades cuya estatura era normal (entre más o menos 0,5 puntaje Z). La evolución del peso y la talla y los antecedentes de desnutrición se obtuvieron de la ficha del consultorio donde estos niños fueron controlados, complementándo- la con los resultados de una evaluación directa mediante balanzas y antropómetros calibrados según recomendaciones internacionales ${ }^{11}$. Se comparó el incremento de estatura en $\mathrm{cm}$ de casos y controles de acuerdo con el estándar OMS. Se construyeron curvas de crecimiento de 0 a 24 meses y de 24 a 72 meses, según la evolución de los puntajes $Z$ del indicador talla edad. Esta división se realizó considerando que el patrón internacional utiliza diferentes técnicas en la medición de los niños y poblaciones de referencia. Se estimaron riesgos relativos para constatar asociaciones de algunas variables con el déficit de crecimiento en estatura y se construyó una tabla de vida de acuerdo a la téenica propuesta por Kaplan y Maier ${ }^{12}$ para comprobar la proporción de des-nutrición en diferentes edades.

Para establecer la significación estadística entre promedios se utilizó análisis de varianza. Se estimaron las ventajas (razones de productos cruzados, OR u "odds ratios") a partir de la formula propuesta por Cornfield para estudios de casos y controles y, para medir significación estadística, el método de chi cuadrado. En la comparación de curvas se utilizó el método de chi cuadrado para tendencia. En todos los casos se consideraron significativos valores de $\mathrm{p}$ inferiores a 0,05 .

\section{Resultados}

El incremento de estatura fue menor en los casos antes y después de los 24 meses ( 5 y 4,5 $\mathrm{cm}$ respectivamente) ( $\mathrm{p}<0,001)$. El aumento observado en los controles fue semejante que el del patrón de referencia (tabla 1 ).

El incremento de la estatura fue menor en los niños casos en todos los intervalos anuales de edad establecidos. La mayor desaceleración ocurrió durante el primer año de vida alcanzando a $3.1 \mathrm{~cm}$; en el segundo año la pérdida fue de $1,9 \mathrm{~cm}$, en el tercero $1,5 \mathrm{~cm}$ y posteriormente se. estabilizó en una pérdida anual de aproximadamente $1 \mathrm{~cm}$. La diferencia con los controles es significativa en todos Ios intervalos (tabla 2). La evolución del indicador talla/edad en puntaje $Z$ de 0 a 18 meses (figura 1), muestra que los ninos casos ya tenían tallas inferiores al nacer $(-0,65$ vs $-0,13)$ y esta siguio disminuyendo progresivamente, Ilegando a $-1,72$ a los 18 me- 
Tabla 1

Incremento de talla acumulado en casos y controles

\begin{tabular}{rcccc}
\hline $\begin{array}{l}\text { Edad } \\
\text { (meses) }\end{array}$ & Casos & Controles & $\begin{array}{c}\text { Inerementa } \\
\text { esperado (cm) }\end{array}$ & p \\
\hline $0-24$ & 31,9 & 35,6 & 36,9 & $<0,001$ \\
$24-72$ & 25,8 & 30,1 & 30,3 & $<0,001$ \\
\hline Total & 57,7 & 65.7 & 67.2 & $<0,001$ \\
\hline
\end{tabular}

\section{Tabla 2}

Incremento de talla según categorías de edad en casos y controles

\begin{tabular}{ccccc}
\hline $\begin{array}{l}\text { Edad } \\
\text { (meses) }\end{array}$ & Casos & Controles & $\begin{array}{c}\text { Increniento } \\
\text { esperadio (cm) }\end{array}$ & p \\
\hline $0-12$ & 21,9 & 24,0 & 25,0 & $<0,001$ \\
$12-24$ & 10,0 & 11,5 & 11.9 & $<0,001$ \\
$24-36$ & 7,9 & 8,9 & 9,4 & $<0,001$ \\
$36-48$ & 6,8 & 7,8 & 7,9 & $<0,001$ \\
$48-60$ & 6,0 & 6,6 & 6,9 & $<0,026$ \\
$60-72$ & 5,1 & 7,0 & 6,2 & $<0,001$ \\
\hline To1al & 57.7 & 65,8 & 67,3 & $<0,001$ \\
& & & &
\end{tabular}

\section{Puntaje Z}

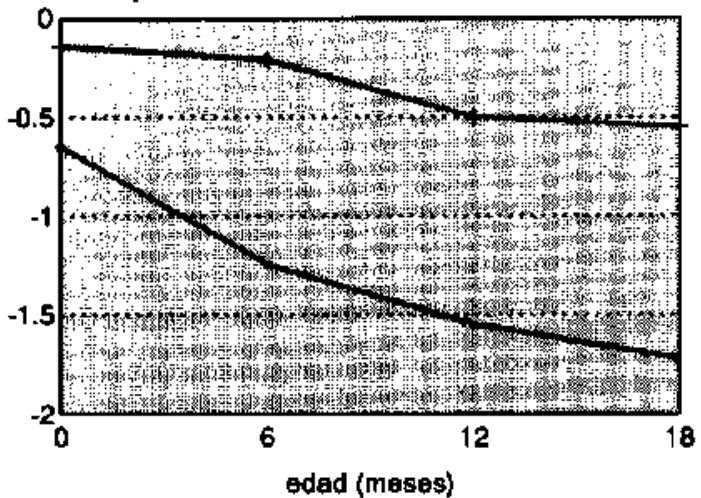

Patrón de referencia NCHSOMS $p<0.001$

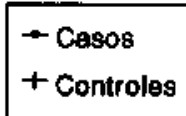

Figura 1: Puntajes $Z$ de talla segín edad en casos y conIroles desde el nacimiento hasta los 18 meses. ses. La pendiente de la curva es más pronunciada durante los primeros 12 meses, especialmente antes de los 6 meses. En cambio la curva de los controles se mantuvo entre la mediana $y$ $\pm 0,5$ puntaje $Z$ durante este período de observacion. Posteriormente el déficit continuó aumentando en los casos, aunque más lentamente, llegando en promedio a $-1,83$ puntaje $Z$ a los 72 meses, mientras los controles persistieron alrededor de la mediana durante este mismo período (figura 2). La distribución de las curvas fue estadísticamente diferente antes y después de los dos años.

El promedio de peso al nacer de los casos fue $3212 \mathrm{~g}$ y el de los controles $3428 \mathrm{~g}$ ( $\mathrm{p}<0,001)$. Las correspondiente tallas de nacimiento fueron $49,1 \mathrm{~cm}$ y $50,3 \mathrm{~cm}(\mathrm{p}<0,001)$.

La ventaja $(O R)$ para tener talla baja en el escolar cuando existe el antecedente de desnutrición alcanza valores muy altos, seguida de la determinada por talla baja al nacer (ambos factores con significación estadística), mientras la del peso de nacimiento insuficiente es cercana a la unidad, no significativa (tabla 3 ).

La proporción de desnutrición (peso/edad $<-1 \mathrm{DE}$ ) aumentó en ambos grupos, siendo su frecuencia más del doble, en todos los intervalos analizados, en los casos que en los controles. Esta mayor proporción ocurrió en ambos grupos en el primer año de vida y fue mínima después

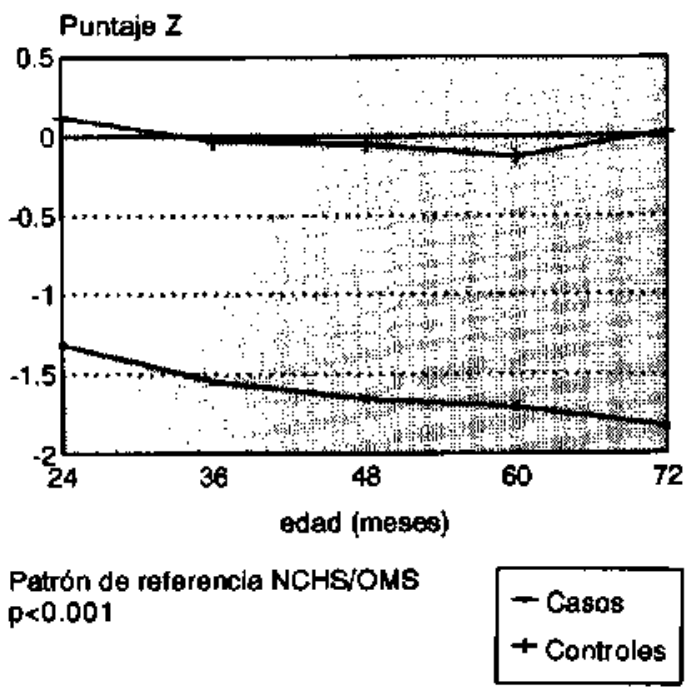

Figura 2: Puntajes $Z$ de talla según edad en casos y controles desde los 24 a 72 meses. 


\section{Tabla 3}

Riesgo de talla baja en escolares según talla baja al nacer, peso insuficiente y desnutrición

\begin{tabular}{lccc}
\hline & $\begin{array}{c}\text { Ventaja } \\
\text { (OR) }\end{array}$ & $\begin{array}{c}\text { Intervalo de } \\
\text { confianza }\end{array}$ & p \\
\hline $\begin{array}{l}\text { Peso de nacimiento } \\
\text { insuficiente }\end{array}$ & 1,30 & $0.51-3,20$ & ns \\
$\begin{array}{l}\text { Talla baja } \\
\text { de nacimiento }\end{array}$ & 3.36 & $1.46 \cdot 7,70$ & 0.001 \\
\begin{tabular}{l} 
Desnutricion \\
\hline
\end{tabular} & 4.94 & $2.63 \cdot 9.25$ & 0,001 \\
\hline
\end{tabular}

Peso de naciniento insuficiente y talla baja al nacer <-I DE. Desnutrición = déficil > -1 DE de pesoledad NCHSTOMS.

de los 19 meses. En la figura 3 se presenta la proporción de niños con desnutrición en distinlas edades, utilizando la técnica de la tabla de vida.

La diferencia de estatura entre los grupos a los seis años fue marcada, alcanzando diferencias superiores a $8 \mathrm{~cm}, y$ fue semejante en niños de uno u otro sexo.

\section{Comentario}

De acuerdo a lo esperado, el incremento de estatura fue menor en los casos que en los controles durante los seis primeros años de vida $y$ el menor aumento ocurrio de preferencia antes de los dos años, aunque la disminución de la velocidad de creciniciento continúa después de esa edad.

El peso promedio de nacimiento fue adecuado en ambos grupos, aunque los casos pesaban menos que los controles. La relevancia de la talla baja al nacer en la determinación de la estatura baja del escolar también ha sido hecha notar en otros estudios recientes ${ }^{13}{ }^{14}$. Su inicio precoz permitiría proponer un componente genético o el efecto de factores ambientales adversos desde la gestación, condiciones que deberían ser motivo de estudios específicos.

El analisis de las curvas de crecimiento permitio objetivar la diferencia entre ambos grupos y el momento del inicio del déficit, que se inicia precozmente y continúa durante los años que

\section{Porcentaje}

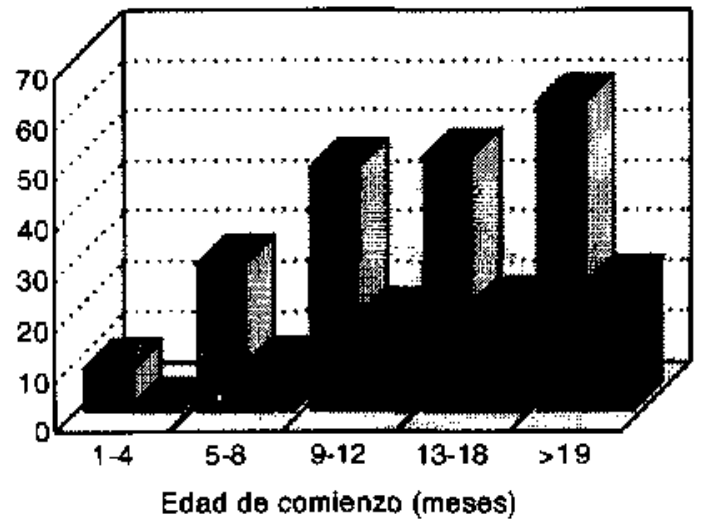

$p<0.0001$

Información obtenlda por Tabla

de Vida de Kaplan-Meier

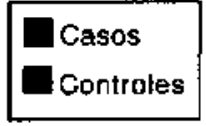

Figura 3: Proporción acumulada de desnutrición según edad de inicio.

preceden el ingreso a la escuela, lo que también ha sido descrito para los niños atendidos en el Sistema Nacional de Servicios de Salud del país y otros niños iberoamericanos ${ }^{15}, 16$.

El aumento progresivo del déficit de talla edad en los casos sugiere el efecto de un componente ambiental, pues si el retardo de crecimiento fuese básicamente de origen genético la curva sería paralela e inferior a la de los niños de estatura normal. Cabe señalar que el déficit antes del primer año no alcanza la magnitud de la talla baja del escolar que ingresa a la enseñanza básica, lo que refuerza la importancia del eventual factor ambiental en la determinacion de la estatura de los escolares chilenos ${ }^{17,14}$. Entre los factores de riesgo estudiados la desnutrición juega un importante papel, al producir una disminución de la multiplicación celular y un retardo de la maduración ósea. La desnutricion produce inicialmente disminución de peso y posteriormente de la velocidad de crecjmiento, hasta su detención si es intensa o prolongada $^{18}$. La disminución de las tasas de desnutrición debería reflejarse en mejoría de la estatura de los niños chilenos, sin embargo los menores que la padecen deben recibir especial cuidado y atención. Aunque en este estudio se excluyeron los niños que presentaban afecciones crónicas, en los casos individuales sería ne- 
cesario considerar el efecto de problemas endocrinos subyacentes no diagnosticados o de retardo constitucional de crecimiento, pues ellos pueden incidir en la talla final del adulto y deberían ser evaluados posteriormente.

La asociación de desnutrición y talla baja al nacer con talla baja del escolar sugiere que ambos criterios deben ser considerados en las acciones de prevención y control prenatales y postnatales dirigidas a cautelar el pleno desarrolio del potencial genético. Entre estas últimas están el seguimiento de madres con riesgo de hijo de talla baja al nacer, lo que hace necesario definir el perfil de ellas e identificarlas, idealmente en atención primaria; el seguimiento pre$\operatorname{coz}$ de los niños con riesgo, la asignación de recursos para la atención integral especializada de desnutridos y la ampliación de la cobertura de intervenciones integrales para lactantes y preescolares.

\section{Referencias}

1. UNICEF: Estado mundial de la infancia. Barceiona: J \& J Asociados. 1995.

2. UNICEF: Una propuesta de clasificación de las comunas del país según la situación de la infancia. Chile: Zenith Lida. 1994.

3. Beaton GH. Bengot SM: Practical population indicalor of health and nutrition. In Nutrition in Preventive Medicine. WHO Monograph Series 1976; 62.

4. Waterlow $J C$ : Reflection on stunting. International Child Health: A digest of Current Information. An Interdational Pediatric Association Publication in collaboration with UNJCEF and WHO 1991; ll (2); 25-35.

5. Martorell R. Rivera J. Kaplonitz H. Pollit E: Long Ierm consequences of growth retadation during early childhood. En: Hernández M, Argente J, ed. Human gtowth: Basic and clinical aspects. Amsterdan, The Netherlands: Elsevier Science Publișhers B.V. 1992: 143-149.

6. Martorell $R$ Rivera $J$. Kaplowit $H$; Consequences of stunting in early childhood for adult body size in rural Guatemala. Ann Nestlé 1990; 48: 85-92.

7. Bustos $P$, Amigo $H$ : Situación nutricional rural: Un estudio en escolares de zonas de alta vulnerabilidad. Rev Chil Pediat 1993: 64: 392.396.

8. Amigo $H$. Bustos $P$ : Factores de riesgo de talla baja en escolanes chilenos de zonas rurales de alta vulnerabilidad social. Arch Lacinoan Nutr 1995: 45: 97-102.

9. Antigo $H$, Bustos $P$, Radrigán $M E$ : Factores de protección de la estatura en escolares rurales de alta vuinerabilidad social. Rev Chil Pediatr 1995; 66: 24-29.

10. OMS: Medición del cambio del estado nutricional: Difectrices para evaluar el efecto nutricional de programas de alimentación suplementaria destinados a grupos vulnerables. Ginebra: OMS 1983.

11. Habikch JP: Estandarización de métodos epidemjologicos cuantitativos sobre el terreno. Bol Of Sanit Panam 1974; $76:$ 375-384.

12. Kaplan EL, Mejer P: No parametric estimation from incomplete observation. J Am Stat Assoc 1958; 53: $457-481$.

13. Ruet MT. Rivera $I$ and Habicht $M P$ : Length screens better than weight in stunted populations. J Nutr 1995; 125: $1222-1228$

14. Amigo $H$, Bustos $P$ : Factotes condicionantes de la estalura en escolares de alta vulnerabilidad social. Santiago: Imptesos Maigret, 1994.

15. Martorell $R$, Rivera $J$. Kaplowitz $H$ : Consecuencias del retraso de crecimiento durante la primera infancia sobre la talla adulta en las zonas rurales de Guatemala. Ann Nestlé 1990; 48: 109-18.

16. Cartillo $C$, Atalah $E$, Ruimalló J, Castro R: Lactancia aarural y estado nutricional del lactante chileno. Bol Of Sanit Panam 1995: $119: 494-502$.

17. De Andraces I, Castillo M. Cortés F: Factores de riesgo para talla baja en escolares de nivel socioeconómico bajo. Rev Chil Pediatr 1994; 65: 303-310.

18. Rus5n P; Aspectos biológicos del desarrollo. En: Meneghello J. Fạnta E, Paris E y Rossejot J. Pediatría, 4* ed. Santiago: Publicaciones Técnicas Mediterróneo 1991. 